\title{
KM3NeT/ARCA sensitivity to transient neutrino sources
}

\author{
Juan Palacios González ${ }^{1, *}$, Marta Colomer Molla ${ }^{1,2}$, Francisco Salesa Greus ${ }^{1}$ \\ and Agustín Sánchez Losa ${ }^{1}$ on behalf of the KM3NeT Collaboration \\ (a complete list of authors can be found at the end of the proceedings) \\ ${ }^{1}$ IFIC - Instituto de Física Corpuscular (CSIC - Universitat de València) \\ c/ Catedrático José Beltrán, 2, 46980 Paterna, Valencia, Spain. \\ ${ }^{2}$ APC, Univ Paris Diderot, CNRS/IN2P3, CEA/Irfu, Obs de Paris, Sorbonne Paris Cité, France. \\ E-mail: Juan.Palacios@ific.uv.es
}

The KM3NeT Collaboration is constructing a $\mathrm{km}^{3}$-volume neutrino telescope in the Mediterranean sea, ARCA (Astroparticle Research with Cosmics in the Abyss), for the detection and subsequent study of high-energy cosmic neutrinos. This telescope will be able to reconstruct the arrival direction of the neutrinos with a precision of $0.1^{\circ}$. The configuration of ARCA makes it sensitive to neutrinos in a wide energy range, from sub-TeV up to tens of PeV. Moreover, this detector has a large field of view and a very high duty cycle, allowing for full-sky (and all-flavours) searches. All these features make ARCA an excellent instrument to study transient neutrino sources.

Atmospheric muons and neutrinos, produced by primary cosmic rays, constitute the main background for ARCA. This background can be several orders of magnitude higher than the expected cosmic neutrino flux. In this work, we introduce an event selection which reduces the background up to a negligible level inside the region of interest and within the search time window. The ARCA performance to detect a transient neutrino flux, including the effective area, sensitivity and discovery potential, are provided for a given test source, and for different time windows.

$37^{\text {th }}$ International Cosmic Ray Conference (ICRC 2021)

July 12th - 23rd, 2021

Online - Berlin, Germany

\footnotetext{
${ }^{*}$ Presenter
} 


\section{Introduction}

Multi-messenger (MM) astronomy is based on the observation of several cosmic messengers coming from the same source. The potential of MM astronomy relies on the fact that a coincident detection of several messengers provides more information than the study of different sources with an unique messenger, and at the same time enhances the detection of faint sources which would not be possible to observe with a single messenger.

Cosmic rays and photons are known cosmic messengers. Both are abundant, however cosmic rays lose their directionality when passing through magnetic fields. Photons are neutral, but at high energies their flux gets attenuated, so only nearby sources can be observed.

In addition to those messengers, the discovery in 2015 of Gravitational Waves from the merger of a binary black hole system [1] marks the beginning of a new era in the study of the Universe.

Finally, cosmic neutrinos, whose existence was first hinted by IceCube in 2013 [2], are another type of messengers that play a very important role in the MM paradigm. As neutrinos are neutral particles, they are not deflected by intergalactic magnetic fields and they point directly towards their origin source. This motivates the development of methods that increase the sensitivity (i.e. the ability of a detector to distinguish signal events from background) to detect neutrinos coming from a specific source. This is of particular interest in the case of transient sources (i.e. with a time-dependent emission), such as Gamma-Ray Bursts and Compact Binary Mergers, since the background in this case is already reduced thanks to the use of narrow time windows. With this analysis, we want to introduce a quick method to derive the sensitivity of ARCA to transient neutrino sources, which will allow this detector to perform a follow-up of the transient event and that can be easily adapted to be used in a partial configuration of the detector.

\section{KM3NeT/ARCA}

KM3NeT ("Cubic Kilometre Neutrino Telescope") is a collaboration which is currently deploying two deep-sea detectors in the Mediterranean Sea. These infrastructures consist of threedimensional arrays of photo-multiplier tubes (PMTs) which are able to detect the Cherenkov light emitted by the particles produced in neutrino interactions on sea water. On the one hand, the low energy array, called ORCA (Oscillation Research with Cosmics in the Abyss) is located $40 \mathrm{~km}$ away from Toulon, and its main objective is the determination of the neutrino mass hierarchy by measuring the atmospheric neutrino oscillations. On the other hand, the high energy array, called ARCA (Astroparticle Research with Cosmic in the Abyss) is located $100 \mathrm{~km}$ away from Portopalo di Capo Passero. The aim of ARCA is the identification, and further study, of the astrophysical sources of high energy neutrinos.

The key element of the KM3NeT detectors is the Digital Optical Module (DOM), a pressureresistant glass sphere where 31 PMTs are embedded. This multi-PMT strategy, in contrast with the use of one single and large PMT in each optical module, has several advantages, such as the ability to recognise physical signals by the coincidence of hits on the same DOM. A total of 18 DOMs are arranged on the same vertical string, called Detection Unit (DU). A set of 115 DUs constitutes a building block (BB), and it is foreseen that ARCA will host two building blocks. Note that both detectors share the same technology and are based on the same Cherenkov detection principle. The 
main difference between them is the density of DOMs, which allows ARCA to cover an energy range which goes from the sub-TeV up to tens of PeV.

\section{Search method}

The search method is based on a binned cut-and-count technique. This means that we define a set of cuts, according to a given optimization criteria, and we study the distribution of the different reconstruction parameters to evaluate the expected number of signal and background events that survive our cuts. The relevant background, in the considered energy range, are atmospheric neutrinos and muons, that we have to properly distinguish from our signal: cosmic neutrinos from a transient source.

A particular flux shape has to be considered for cosmic and atmospheric neutrinos in order to perform Monte Carlo (MC) simulations. For atmospheric neutrinos, the conventional flux of the model Honda2006 has been used [3]. A correction for the knee (of the cosmic-ray spectrum) has been included according to the Gaisser-H3a model [4]. For cosmic neutrinos, a power law $\Phi=\Phi_{0} E^{-\gamma}$ has been considered, with $\Phi_{0}=10^{-9} \mathrm{GeV}^{-1} \mathrm{~cm}^{-2} \mathrm{~s}^{-1} \mathrm{sr}^{-1}$ and $\gamma=2.0$. We will work under the assumption that all the signal flux for the cosmic model is emitted within the given time window, as we are dealing with transient sources.

A time window of 1000 seconds is used for a test source considered in this analysis. The reason for this particular selection is that it is a common choice between neutrino telescopes to perform the follow-up of Gravitational Waves events and Gamma Ray Bursts. In the case of the test source, we will consider that it is located at a zenith of $70^{\circ}$ and an azimuth of $300^{\circ}$ in local coordinates, just as a reference point in the upgoing sky.

The neutrino flavour identification in large volume neutrino telescopes such as ARCA is not possible. However, two different event topologies can be distinguished in these kind of detectors using the hit pattern. On the one hand, track-like events are those in which the hit pattern is consistent with a straight line. This feature is characteristic of the pathway of muons in water, so we relate this topology with $v_{\mu}$ CC interactions. On the other hand, shower-like events are those events in which the hit pattern can be seen as the quasi-spherical light emission from a localised point. This pattern can be related with electromagnetic and hadronic cascades of electrons and hadrons in water, and therefore is associated with $v_{e} \mathrm{CC}$ events and $\mathrm{NC}$ interactions of all neutrino flavors.

Another remarkable point is the distinction between upgoing and downgoing events. Upgoing events are defined as those who travel across the Earth in their way to the detector. Selecting these events is very useful to reject atmospheric muons, since only neutrinos can cross the Earth. The present analysis has been focused on a sample of upgoing tracks, since these are the main interaction channel to which neutrino telescopes are sensitive. However, the prospect is that this follow-up method can be extended not only to downgoing events but also to include showers.

The reconstruction of the simulated MC events has been performed with the official KM3NeT reconstruction algorithm for tracks [5]. In particular, for each event, the interaction vertex, the particle energy, the direction of the outgoing lepton and the track length are reconstructed. The quality parameter of the reconstruction (likelihood) is also provided for each event. Table (1) shows the reconstruction variables that have been used in this analysis, which are the ones to which we will apply our selection cuts. 


\begin{tabular}{|c|c|}
\hline Variable & Description \\
\hline Zenith angle & Angle between the zenith of the upgoing sky and the reconstruction direction \\
\hline$\beta_{0}$ & Angular error estimation of the event \\
\hline Length & Distance (in meters) between the first and last hits \\
\hline Likelihood & Likelihood of the reconstruction (quality parameter) \\
\hline
\end{tabular}

Table 1: Variables of the reconstructed algorithm used in this analysis

The Region of Interest (RoI) is defined as the sky region selected to search for space coincidences with an identified signal. It is given, for each event, by the angle between the reconstructed direction and the direction of the source, defining a cone for the search. Only events which are reconstructed close enough to the source (i.e. inside the RoI) are considered.

\subsection{Optimization procedure}

The optimization procedure can be divided into two parts: the determination of the pre-cuts and the optimization of the cuts. First, the pre-cuts are set in order to reduce as much as possible both the background and the shower contamination of our sample, as we want a pure track sample. Shower events, when reconstructed by the track reconstruction algorithm, are expected to have some characteristic attributes, such as large angular errors $\beta_{0}$ and short track lengths. According to these features, we will use these two variables to optimise the pre-cuts.

Our pure track sample is defined as a neutrino sample with a shower contamination smaller than a $1 \%$ after applying the selection. Since several pairs of cuts in $\log _{10} \beta_{0}$ and length satisfy this condition, we choose the optimal set of cuts as the one maximizing the number of pure-track signal events. The pre-cuts obtained with this strategy are found to reduce also the background contamination by three orders of magnitude with respect to the signal.

Once the pre-cuts are determined, the next step is the optimization of the rest of the variables: the likelihood and RoI radius. This optimization will be performed by minimizing the Model Rejection Factor (MRF) [6], which is defined as

$$
\mathrm{MRF}=\frac{\bar{\mu}_{90}\left(n_{b}\right)}{n_{s}}, \quad \text { with } \quad \bar{\mu}_{90}\left(n_{b}\right)=\sum_{n_{\mathrm{obs}}=0}^{\infty} \mu_{90}^{\mathrm{FC}}\left(n_{\mathrm{obs}}, n_{b}\right) \frac{n_{b}^{n_{\mathrm{obs}}} e^{-n_{b}}}{n_{\mathrm{obs}} !}
$$

where $n_{s}\left(n_{b}\right)$ denote the expected number of signal (background) events that will survive our cuts, respectively, and $\mu_{90}^{\mathrm{FC}}\left(n_{\mathrm{obs}}, n_{b}\right)$ denotes the $90 \% \mathrm{CL}$ upper limit derived using the Feldman-Cousins (FC) approach [7]. This parameter depends on the observed number of events once the experiment is performed. Since we are dealing with a MC, we have to replace this limit by the average (or expected) upper limit $\bar{\mu}_{90}\left(n_{b}\right)$, which is defined as the sum of the FC upper limits for all the possible $n_{\mathrm{obs}}$, weighted by the Poisson probability.

\section{Results}

For the determination of the pre-cuts, the computation of the shower contamination and the fraction of track signal that survives has been performed according to the procedure explained in 
section 3.1. The calculation carried out indicated that with the cuts $\log _{10} \beta_{0}<-0.7$ and track length $>340 \mathrm{~m}$, the shower contamination in the sample was reduced to $0.94 \%$ and the fraction of track signal that survived was $72 \%$. The energy distribution of the events once these pre-cuts are applied is shown in figure (1). The median angular resolution of the sample is $0.3^{\circ}$ once the pre-cuts are applied. Note that background (both muons and atmospheric neutrinos) are especially reduced for this particular selection. Therefore, the pre-cuts not only provide us with a clean sample of track events, but also reduce the background by one order of magnitude with respect to the signal. This background will be reduced even more once the RoI is applied.

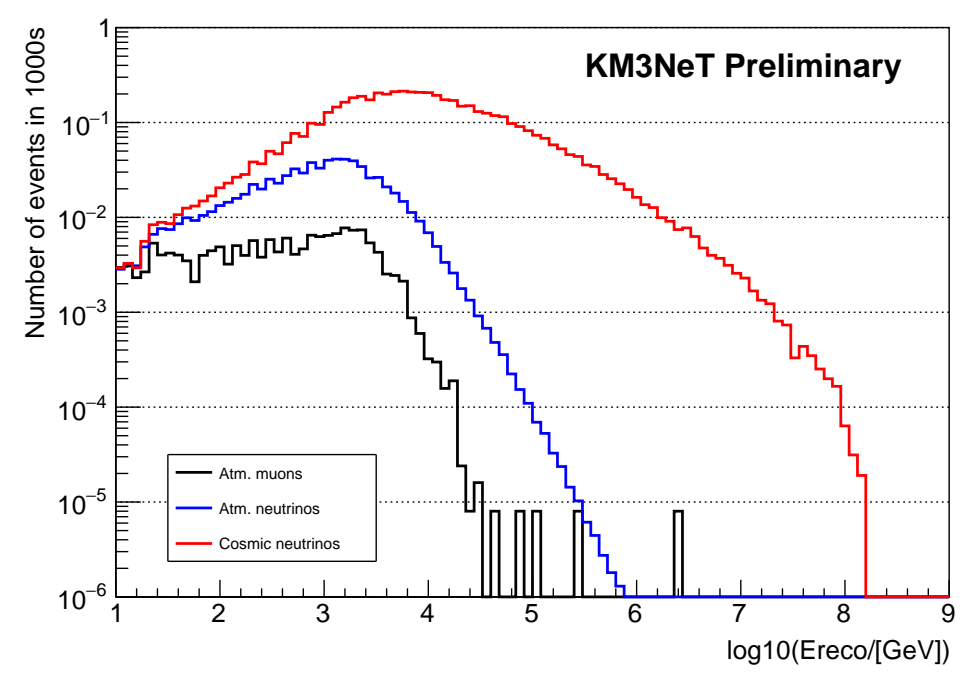

Figure 1: Monte-Carlo energy distribution of cosmic neutrinos, atmospheric neutrinos and atmospheric muons once the pre-cuts have been applied. Note that atmospheric muons are orders of magnitude below of the signal.

Once the pre-cuts have been determined, the computation of the MRF for different cuts in the likelihood and different RoI radii has been performed. The optimum RoI radius is large enough to contain as much signal as possible, but with the condition that if we increase this radius we would only gain more background without changing the signal appreciably. The values obtained indicated that the optimum RoI radius is found at $6.5^{\circ}$. For the case of the likelihood, we find that the MRF is not sensitive to cuts in such variable, which indicates that there is no need for applying a cut on the likelihood. This has sense since, as can be seen in table (2), the background is three orders of magnitude below the signal once the RoI is applied, so the optimization procedure does not find relevant changes when including a cut in the likelihood.

The same procedure can be repeated for different time windows. We provide the results according to this method also for 1 day and 10 days. The pre-cuts used are the same in the three cases, since the shower contamination is not affected by the time window.

Once the analysis selection is defined, one aims to derive which are the ARCA capabilities concerning the detection of a transient neutrino signal. Here, we present the results in terms of the fluence sensitivity and the discovery potential.

The fluence sensitivity is defined as the time integrated neutrino energy flux. This can be done 


\begin{tabular}{|c|c|c|c||c|}
\cline { 2 - 5 } \multicolumn{1}{c|}{} & Upgoing cut & $+\log _{10} \beta_{0}<-0.7$ & + length $>340 \mathrm{~m}$ & RoI $\left(6.5^{\circ}\right)$ \\
\hline Cosmic neutrinos & 10.4 & 6.4 & 5.4 & 5.3 \\
\hline Atm. neutrinos & 1.4 & 0.79 & 0.74 & $5.1 \cdot 10^{-3}$ \\
\hline Atm. muons & 3.4 & 0.21 & 0.16 & $0.96 \cdot 10^{-3}$ \\
\hline
\end{tabular}

Table 2: Expected number of events for $1000 \mathrm{~s}$ of operation of one BB of ARCA, for a source located at $\left(70^{\circ}, 300^{\circ}\right)$.

by means of the acceptance, which is defined as the integral in energy and time of the effective area convoluted by the normalized flux:

$$
A_{c c}=\int \mathrm{d} t \int \mathrm{d} E_{v} A_{e f f}\left(E_{v}\right) E_{v}^{-\gamma}
$$

The effective area is defined as the equivalent surface which is $100 \%$ efficient and detects the same number of events as the detector, for a particle beam perpendicular to that surface. Figure (2) provides the effective area of ARCA once our pre-cuts have been applied.

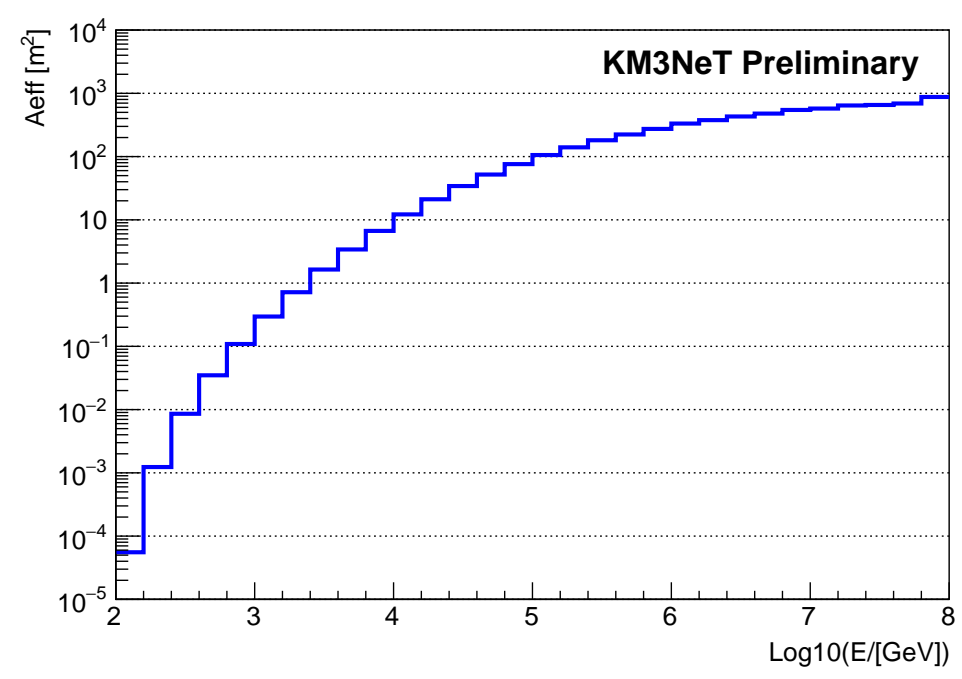

Figure 2: Effective area of ARCA (2 BB) after the pre-cuts.

The expected number of signal events is related to the acceptance through the flux normalization factor $\Phi_{0}$. This allows to obtain the fluence sensitivity as the average FC upper limit divided by the acceptance. Figure (3) shows the results obtained for the sensitivity as a function of the energy.

The discovery potential, which is defined as the number of signal events that are needed to be emitted from the source for an experiment to claim a discovery at a given significance [8], has been computed for the background events remaining inside the RoIs provided by the MRF method. The results are given in table (3). The integrated sensitivity, which is the fluence sensitivity integrated in the energy range $10^{3}-10^{7} \mathrm{GeV}$, is also provided. 


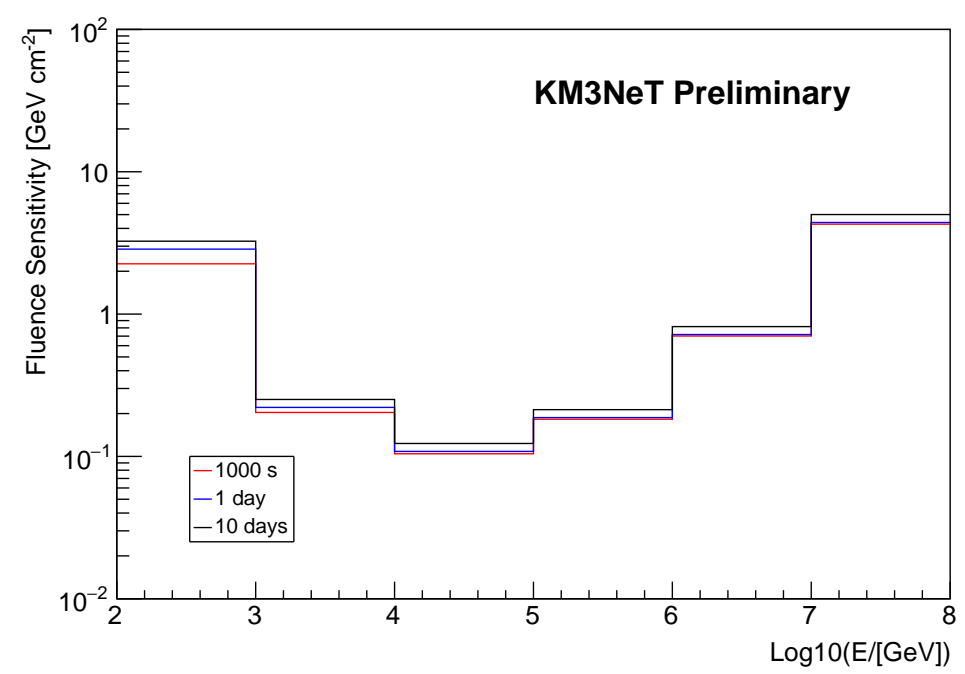

Figure 3: Differential fluence sensitivity as a function of the neutrino energy for the two full BB of the ARCA detector, for the three different time windows considered in this analysis.

\begin{tabular}{|c|c|c|c|c|}
\hline $\begin{array}{c}\text { Time } \\
\text { window }\end{array}$ & $\begin{array}{c}\text { Optimum } \\
\text { RoI radius MRF }\end{array}$ & $\begin{array}{c}\text { Expected } \\
\text { background events }\end{array}$ & $\begin{array}{c}\text { Fluence } \\
\text { sensitivity } \\
\left(\mathrm{GeV} \cdot \mathrm{cm}^{-2}\right)\end{array}$ & $\begin{array}{c}\text { Discovery } \\
N_{\mathrm{sg}}^{5 \sigma}(50 \%)\end{array}$ \\
\hline $1000 \mathrm{~s}$ & $6.5^{\circ}$ & $1.2 \cdot 10^{-2}$ & 0.047 & 2.7 \\
\hline 1 day & $3.0^{\circ}$ & $3.3 \cdot 10^{-2}$ & 0.050 & 3.6 \\
\hline 10 days & $2.0^{\circ}$ & $1.2 \cdot 10^{-1}$ & 0.061 & 4.6 \\
\hline
\end{tabular}

Table 3: Sensitivity and discovery values for the optimum RoI radius obtained using the MRF, for the different time windows. The expected numbers of background events are given for 2 BB of ARCA.

\section{Conclusions}

An optimum set of pre-cuts has been derived in this work to increase the sensitivity of ARCA to transient sources. A reduction of the background is achieved using the pre-cuts and the RoI radii obtained for the cases of a time window of 1000 seconds, one day and ten days. We find that the optimum size of the RoI according to the MRF method is reduced as we increase the time window. This is expected since as we enlarge the time window, the background is increased too, so the optimization procedure provides a smaller RoI radius.

The resulting fluence sensitivity can be compared with similar results from other large-volume neutrino telescopes. In the case of ANTARES [9], for a time window of $1000 \mathrm{~s}$, an average fluence upper limit of the order of magnitude of $\sim 0.8-1.4 \mathrm{GeV} \mathrm{cm}^{-2}$ is found for the upgoing sky in the energy range from $\sim 3 \mathrm{TeV}$ to $\sim 3 \mathrm{PeV}$. This limit is two orders of magnitude less stringent than the result presented here. In the case of IceCube [10], the results for a time window of $1000 \mathrm{~s}$ vary from 0.03 to $0.7 \mathrm{GeV} \mathrm{cm}^{-2}$ depending on the source considered, and both for the upgoing and downgoing sky in the energy range $\sim 10 \mathrm{TeV}$ to $\sim 100 \mathrm{PeV}$. Only the order of magnitude of the fluence sensitivity can be compared since the ANTARES and IceCube analyses are for extended 
sources, while our result can be considered as a point-source result. The sensitivity obtained with this binned cut-and-count method has a similar order of magnitude to the best IceCube results in similar analyses of this field, in a broader energy range.

\section{Acknowledgments}

This work has been supported by Ministerio de Ciencia, Innovación, Investigación y Universidades (MCIU): Programa Estatal de Generación de Conocimiento (refs. PGC2018-096663B-C41) (MCIU/FEDER), and Generalitat Valenciana: GenT (ref. CIDEGENT/2018/034 and CIDEGENT/2020/04).

\section{References}

[1] LIGO ScIENTIFIC, VIRGO collaboration, Observation of Gravitational Waves from a Binary Black Hole Merger, Phys. Rev. Lett. 116 (2016) 061102 [1602 . 03837].

[2] ICECuBE collaboration, Evidence for High-Energy Extraterrestrial Neutrinos at the IceCube Detector, Science 342 (2013) 1242856 [1311. 5238].

[3] M. Honda, T. Kajita, K. Kasahara, S. Midorikawa and T. Sanuki, Calculation of atmospheric neutrino flux using the interaction model calibrated with atmospheric muon data, Phys. Rev. D 75 (2007) 043006 [astro-ph/0611418].

[4] T.K. Gaisser, Spectrum of cosmic-ray nucleons, kaon production, and the atmospheric muon charge ratio, Astropart. Phys. 35 (2012) 801 [1111.6675].

[5] KM3NET collaboration, KM3NeT/ARCA Event Reconstruction Algorithms, PoS ICRC2017 (2018) 950.

[6] G.C. Hill and K. Rawlins, Unbiased cut selection for optimal upper limits in neutrino detectors: The Model rejection potential technique, Astropart. Phys. 19 (2003) 393 [astro-ph/0209350].

[7] G.J. Feldman and R.D. Cousins, A Unified approach to the classical statistical analysis of small signals, Phys. Rev. D 57 (1998) 3873 [physics/9711021].

[8] G.C. Hill, J. Hodges, B. Hughey, A. Karle and M. Stamatikos, Examining the balance between optimising an analysis for best limit setting and best discovery potential, in Statistical Problems in Particle Physics, Astrophysics and Cosmology, 9, 2005.

[9] ANTARES collaboration, Search for neutrino counterparts of gravitational-wave events detected by LIGO and Virgo during run O2 with the ANTARES telescope, Eur. Phys. J. C 80 (2020) 487 [2003.04022].

[10] ICECUBE collaboration, IceCube Search for Neutrinos Coincident with Compact Binary Mergers from LIGO-Virgo's First Gravitational-wave Transient Catalog, Astrophys. J. Lett. 898 (2020) L10 [2004. 02910]. 


\section{Full Author List: KM3NeT Collaboration}

M. Ageron ${ }^{1}$, S. Aiello ${ }^{2}$, A. Albert ${ }^{3,55}$, M. Alshamsi ${ }^{4}$, S. Alves Garre ${ }^{5}$, Z. Aly ${ }^{1}$, A. Ambrosone ${ }^{6,7}$, F. Ameli ${ }^{8}$, M. Andre 9 , G. Androulakis ${ }^{10}$, M. Anghinolfi ${ }^{11}$, M. Anguita ${ }^{12}$, G. Anton ${ }^{13}$, M. Ardid ${ }^{14}$, S. $\operatorname{Ardid}^{14}$, W. Assal ${ }^{1}$, J. Aublin ${ }^{4}$, C. Bagatelas ${ }^{10}$, B. Baret ${ }^{4}$, S. Basegmez du Pree ${ }^{15}$, M. Bendahman ${ }^{4,16}$, F. Benfenati ${ }^{17,18}$, E. Berbee ${ }^{15}$, A. M. van den Berg ${ }^{19}$, V. Bertin ${ }^{1}$, S. Beurthey ${ }^{1}$, V. van Beveren ${ }^{15}$, S. Biagi ${ }^{20}$, M. Billault ${ }^{1}$, M. Bissinger ${ }^{13}$, M. Boettcher ${ }^{21}$, M. Bou Cabo ${ }^{22}$, J. Boumaaza ${ }^{16}$, M. Bouta ${ }^{23}$, C. Boutonnet ${ }^{4}$, G. Bouvet ${ }^{24}$, M. Bouwhuis ${ }^{15}$, C. Bozza ${ }^{25}$, H.Brânzaş ${ }^{26}$, R. Bruijn ${ }^{15,27}$, J. Brunner ${ }^{1}$, R. Bruno ${ }^{2}$, E. Buis ${ }^{28}$, R. Buompane ${ }^{6,29}$, J. Busto ${ }^{1}$, B. Caiffi ${ }^{11}$, L. Caillat ${ }^{1}$, D. Calvo ${ }^{5}$, S. Campion ${ }^{30,8}$, A. Capone ${ }^{30,8}$, H. Carduner ${ }^{24}$, V. Carretero ${ }^{5}$, P. Castaldi ${ }^{17,31}$, S. Celli ${ }^{30,8}$, R. Cereseto ${ }^{11}$, M. Chabab ${ }^{32}$, C. Champion ${ }^{4}$, N. $\mathrm{Chau}^{4}$, A. Chen ${ }^{33}$, S. Cherubinini ${ }^{20,34}$, V. Chiarella ${ }^{35}$, T. Chiarusi ${ }^{17}$, M. Circella ${ }^{36}$, R. Cocimano ${ }^{20}$, J. A. B. Coelho ${ }^{4}$, A. Coleiro ${ }^{4}$, M. Colomer Molla ${ }^{4,5}$, S. Colonges ${ }^{4}$, R. Coniglione ${ }^{20}$, A. Cosquer ${ }^{1}$, P. Coyle ${ }^{1}$, M. Cresta ${ }^{11}$, A. Creusot ${ }^{4}$, A. $\mathrm{Cruz}^{37}$, G. Cuttone ${ }^{20}$, A. D’Amico ${ }^{15}$, R. Dallier ${ }^{24}$, B. De Martino ${ }^{1}$, M. De Palma ${ }^{36,38}$, I. Di Palma ${ }^{30,8}$, A. F. Díaz ${ }^{12}$, D. Diego$\operatorname{Tortosa}^{14}$, C. Distefano ${ }^{20}$, A. Domi ${ }^{15,27}$, C. Donzaud ${ }^{4}$, D. Dornic ${ }^{1}$, M. Dörr ${ }^{39}$, D. Drouhin ${ }^{3,55}$, T. Eberl ${ }^{13}$, A. Eddyamoui ${ }^{16}$, T. van Eeden ${ }^{15}$, D. van Eijk ${ }^{15}$, I. El Bojaddaini ${ }^{23}$, H. Eljarrari ${ }^{16}$, D. Elsaesser ${ }^{39}$, A. Enzenhöfer ${ }^{1}$, V. Espinosa ${ }^{14}$, P. Fermani ${ }^{30,8}$, G. Ferrara ${ }^{20,34}$, M. D. Filipović ${ }^{40}$, F. Filippini ${ }^{17,18}$, J. Fransen ${ }^{15}$, L. A. Fusco ${ }^{1}$, D. Gajanana ${ }^{15}$, T. Gal ${ }^{13}$, J. García Méndez ${ }^{14}$, A. Garcia Soto $^{5}$, E. Garçon ${ }^{1}$, F. Garufi ${ }^{6,7}$, C. Gatius ${ }^{15}$, N. Geißelbrecht ${ }^{13}$, L. Gialanella ${ }^{6,29}$, E. Giorgio ${ }^{20}$, S. R. Gozzini ${ }^{5}$, R. Gracia ${ }^{15}$, K. Graf ${ }^{13}$, G. Grella ${ }^{41}$, D. Guderian ${ }^{56}$, C. Guidi ${ }^{11,42}$, B. Guillon ${ }^{43}$, M. Gutiérrez ${ }^{44}$, J. Haefner ${ }^{13}$, S. Hallmann ${ }^{13}$, H. Hamdaoui ${ }^{16}$, H. van Haren ${ }^{45}$, A. Heijboer ${ }^{15}$, A. Hekalo ${ }^{39}$, L. Hennig ${ }^{13}$, S. Henry ${ }^{1}$, J. J. Hernández-Rey ${ }^{5}$, J. Hofestädt ${ }^{13}$, F. Huang ${ }^{1}$, W. Idrissi Ibnsalih ${ }^{6,29}$, A. Ilioni ${ }^{4}$, G. Illuminati ${ }^{17,18,4}$, C. W. James ${ }^{37}$, D. Janezashvili ${ }^{46}$, P. Jansweijer ${ }^{15}$, M. de Jong ${ }^{15,47}$, P. de Jong ${ }^{15,27}$, B. J. Jung ${ }^{15}$, M. Kadler ${ }^{39}$, P. Kalaczyński ${ }^{48}$, O. Kalekin ${ }^{13}$, U. F. Katz ${ }^{13}$, F. Kayzel $^{15}$, P. Keller ${ }^{1}$, N. R. Khan Chowdhury ${ }^{5}$, G. Kistauri ${ }^{46}$, F. van der Knaap ${ }^{28}$, P. Kooijman ${ }^{27,57}$, A. Kouchner ${ }^{4,49}$, M. Kreter ${ }^{21}$, V. Kulikovskiy ${ }^{11}$, M. Labalme ${ }^{43}$, P. Lagier ${ }^{1}$, R. Lahmann ${ }^{13}$, P. Lamare ${ }^{1}$, M. Lamoureux ${ }^{14}$, G. Larosa ${ }^{20}$, C. Lastoria ${ }^{1}$, J. Laurence ${ }^{1}$, A. Lazo $^{5}$, R. Le Breton ${ }^{4}$, E. Le Guirriec ${ }^{1}$, S. Le Stum ${ }^{1}$, G. Lehaut ${ }^{43}$, O. Leonardi ${ }^{20}$, F. Leone ${ }^{20,34}$, E. Leonora ${ }^{2}$, C. Lerouvillois ${ }^{1}$, J. Lesrel ${ }^{4}$, N. Lessing ${ }^{13}$, G. Levi ${ }^{17,18}$, M. Lincetto ${ }^{1}$, M. Lindsey Clark ${ }^{4}$, T. Lipreau ${ }^{24}$, C. LLorens Alvarez ${ }^{14}$, A. Lonardo ${ }^{8}$, F. Longhitano ${ }^{2}$, D. Lopez-Coto ${ }^{44}$, N. Lumb ${ }^{1}$, L. Maderer ${ }^{4}$, J. Majumdar ${ }^{15}$, J. Mańczak ${ }^{5}$, A. Margiotta ${ }^{17,18}$, A. Marinelli ${ }^{6}$, A. Marini ${ }^{1}$, C. Markou $^{10}$, L. Martin ${ }^{24}$, J. A. Martínez-Mora ${ }^{14}$, A. Martini ${ }^{35}$, F. Marzaioli ${ }^{6,29}$,

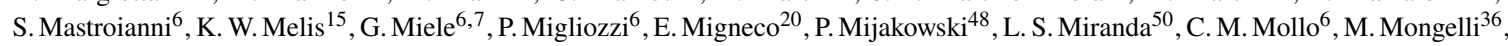
A. Moussa ${ }^{23}$, R. Muller ${ }^{15}$, P. Musico ${ }^{11}$, M. Musumeci ${ }^{20}$, L. Nauta ${ }^{15}$, S. Navas ${ }^{44}$, C. A. Nicolau ${ }^{8}$, B. Nkosi ${ }^{33}$, B. Ó Fearraigh ${ }^{15,27}$, M. O'Sullivan ${ }^{37}$, A. Orlando ${ }^{20}$, G. Ottonello ${ }^{11}$, S. Ottonello ${ }^{11}$, J. Palacios González ${ }^{5}$, G. Papalashviliite, R. Papaleo ${ }^{20}$, C. Pastore ${ }^{36}$, A. M. Păun ${ }^{26}$, G.E. Păvălaşş, G. Pellegrinini ${ }^{17}$, C. Pellegrino ${ }^{18,58}$, M. Perrin-Terrinin ${ }^{1}$, V. Pestel ${ }^{15}$, P. Piattelli ${ }^{20}$, C. Pieterse ${ }^{5}$, O. Pisanti ${ }^{6,7}$, C. Poirè ${ }^{14}$, V. Popa $^{26}$, T. Pradier $^{3}$, F. Pratolongo ${ }^{11}$, I. Probst ${ }^{13}$, G. Pühlhofer ${ }^{51}$, S. Pulvirenti ${ }^{20}$, G. Quéméner ${ }^{43}$, N. Randazzo ${ }^{2}$, A. Rapicavoli ${ }^{34}$, S. Razzaque $^{50}$, D. Real ${ }^{5}$, S. Reck ${ }^{13}$, G. Riccobene ${ }^{20}$, L. Rigalleau ${ }^{24}$, A. Romanov ${ }^{11,42}$, A. Rovelli ${ }^{20}$, J. Royon ${ }^{1}$, F. Salesa Greus ${ }^{5}$, D. F. E. Samtleben ${ }^{15,47}$, A. Sánchez Losa ${ }^{36,5}$, M. Sanguineti ${ }^{11,42}$, A. Santangelo ${ }^{51}$, D. Santonocito ${ }^{20}$, P. Sapienza ${ }^{20}$, J. Schmelling ${ }^{15}$, J. Schnabel ${ }^{13}$, M. F. Schneider ${ }^{13}$, J. Schumann ${ }^{13}$, H. M. Schutte ${ }^{21}$, J. Seneca ${ }^{15}$, I. Sgura ${ }^{36}$, R. Shanidze ${ }^{46}$, A. Sharma ${ }^{52}$, A. Sinopoulou ${ }^{10}$, B. Spisso ${ }^{41,6}$, M. Spurio ${ }^{17,18}$, D. Stavropoulos ${ }^{10}$, J. Steijger ${ }^{15}$, S. M. Stellacci ${ }^{41,6}$, M. Taiuti ${ }^{11,42}$, F. Tatone ${ }^{36}$, Y. Tayalati ${ }^{16}$, E. Tenllado ${ }^{44}$, D. Tézier ${ }^{1}$, T. Thakore ${ }^{5}$, S. Theraube ${ }^{1}$, H. Thiersen $^{21}$, P. Timmer $^{15}$, S. Tingay ${ }^{37}$, S. Tsagkli $^{10}$, V. Tsourapis ${ }^{10}$, E. Tzamariudaki ${ }^{10}$, D. Tzanetatos ${ }^{10}$, C. Valieri ${ }^{17}$, V. Van Elewyck ${ }^{4,49}$, G. Vasileiadis ${ }^{53}$, F. Versari ${ }^{17,18}$, S. Viola $^{20}$, D. Vivolo ${ }^{6,29}$, G. de Wasseige ${ }^{4}$, J. Wilms ${ }^{54}$, R. Wojaczyński ${ }^{48}$, E. de Wolf ${ }^{15,27}$, T. Yousfi ${ }^{23}$, S. Zavatarelli ${ }^{11}$, A. Zegarelli ${ }^{30,8}$, D. Zito ${ }^{20}$, J. D. Zornoza ${ }^{5}$, J. Zúñiga ${ }^{5}$, N. Zywucka ${ }^{21}$.

${ }^{1}$ Aix Marseille Univ, CNRS/IN2P3, CPPM, Marseille, France.

${ }^{2}$ INFN, Sezione di Catania, Via Santa Sofia 64, Catania, 95123 Italy.

${ }^{3}$ Université de Strasbourg, CNRS, IPHC UMR 7178, F-67000 Strasbourg, France.

${ }^{4}$ Université de Paris, CNRS, Astroparticule et Cosmologie, F-75013 Paris, France.

${ }^{5}$ IFIC - Instituto de Física Corpuscular (CSIC - Universitat de València), c/Catedrático José Beltrán, 2, 46980 Paterna, Valencia, Spain. ${ }^{6}$ INFN, Sezione di Napoli, Complesso Universitario di Monte S. Angelo, Via Cintia ed. G, Napoli, 80126 Italy.

${ }^{7}$ Università di Napoli “Federico II", Dip. Scienze Fisiche "E. Pancini”, Complesso Universitario di Monte S. Angelo, Via Cintia ed. G, Napoli, 80126 Italy.

${ }^{8}$ INFN, Sezione di Roma, Piazzale Aldo Moro 2, Roma, 00185 Italy.

${ }^{9}$ Universitat Politècnica de Catalunya, Laboratori d'Aplicacions Bioacústiques, Centre Tecnològic de Vilanova i la Geltrú, Avda. Rambla Exposició, s/n, Vilanova i la Geltrú, 08800 Spain.

${ }^{10}$ NCSR Demokritos, Institute of Nuclear and Particle Physics, Ag. Paraskevi Attikis, Athens, 15310 Greece.

${ }^{11}$ INFN, Sezione di Genova, Via Dodecaneso 33, Genova, 16146 Italy.

${ }^{12}$ University of Granada, Dept. of Computer Architecture and Technology/CITIC, 18071 Granada, Spain.

${ }^{13}$ Friedrich-Alexander-Universität Erlangen-Nürnberg, Erlangen Centre for Astroparticle Physics, Erwin-Rommel-Straße 1, 91058 Erlangen, Germany.

${ }^{14}$ Universitat Politècnica de València, Instituto de Investigación para la Gestión Integrada de las Zonas Costeras, C/ Paranimf, 1, Gandia, 46730 Spain.

${ }^{15}$ Nikhef, National Institute for Subatomic Physics, PO Box 41882, Amsterdam, 1009 DB Netherlands.

${ }^{16}$ University Mohammed V in Rabat, Faculty of Sciences, 4 av. Ibn Battouta, B.P. 1014, R.P. 10000 Rabat, Morocco.

${ }^{17}$ INFN, Sezione di Bologna, v.le C. Berti-Pichat, 6/2, Bologna, 40127 Italy.

\footnotetext{
${ }^{1}$ also at Dipartimento di Fisica, INFN Sezione di Padova and Università di Padova, I-35131, Padova, Italy
} 
${ }^{18}$ Università di Bologna, Dipartimento di Fisica e Astronomia, v.le C. Berti-Pichat, 6/2, Bologna, 40127 Italy.

${ }^{19}$ KVI-CART University of Groningen, Groningen, the Netherlands.

${ }^{20}$ INFN, Laboratori Nazionali del Sud, Via S. Sofia 62, Catania, 95123 Italy.

${ }^{21}$ North-West University, Centre for Space Research, Private Bag X6001, Potchefstroom, 2520 South Africa.

${ }^{22}$ Instituto Español de Oceanografía, Unidad Mixta IEO-UPV, C/ Paranimf, 1, Gandia, 46730 Spain.

${ }^{23}$ University Mohammed I, Faculty of Sciences, BV Mohammed VI, B.P. 717, R.P. 60000 Oujda, Morocco.

${ }^{24}$ Subatech, IMT Atlantique, IN2P3-CNRS, Université de Nantes, 4 rue Alfred Kastler - La Chantrerie, Nantes, BP 2072244307 France.

${ }^{25}$ Università di Salerno e INFN Gruppo Collegato di Salerno, Dipartimento di Matematica, Via Giovanni Paolo II 132, Fisciano, 84084 Italy.

${ }^{26}$ ISS, Atomistilor 409, Măgurele, RO-077125 Romania.

${ }^{27}$ University of Amsterdam, Institute of Physics/IHEF, PO Box 94216, Amsterdam, 1090 GE Netherlands.

${ }^{28}$ TNO, Technical Sciences, PO Box 155, Delft, 2600 AD Netherlands.

${ }^{29}$ Università degli Studi della Campania "Luigi Vanvitelli", Dipartimento di Matematica e Fisica, viale Lincoln 5, Caserta, 81100 Italy.

${ }^{30}$ Università La Sapienza, Dipartimento di Fisica, Piazzale Aldo Moro 2, Roma, 00185 Italy.

${ }^{31}$ Università di Bologna, Dipartimento di Ingegneria dell'Energia Elettrica e dell'Informazione "Guglielmo Marconi", Via dell’Università 50, Cesena, 47521 Italia.

${ }^{32}$ Cadi Ayyad University, Physics Department, Faculty of Science Semlalia, Av. My Abdellah, P.O.B. 2390, Marrakech, 40000 Morocco.

${ }^{33}$ University of the Witwatersrand, School of Physics, Private Bag 3, Johannesburg, Wits 2050 South Africa.

${ }^{34}$ Università di Catania, Dipartimento di Fisica e Astronomia "Ettore Majorana", Via Santa Sofia 64, Catania, 95123 Italy.

${ }^{35}$ INFN, LNF, Via Enrico Fermi, 40, Frascati, 00044 Italy.

${ }^{36}$ INFN, Sezione di Bari, via Orabona, 4, Bari, 70125 Italy.

${ }^{37}$ International Centre for Radio Astronomy Research, Curtin University, Bentley, WA 6102, Australia.

${ }^{38}$ University of Bari, Via Amendola 173, Bari, 70126 Italy.

${ }^{39}$ University Würzburg, Emil-Fischer-Straße 31, Würzburg, 97074 Germany.

${ }^{40}$ Western Sydney University, School of Computing, Engineering and Mathematics, Locked Bag 1797, Penrith, NSW 2751 Australia.

${ }^{41}$ Università di Salerno e INFN Gruppo Collegato di Salerno, Dipartimento di Fisica, Via Giovanni Paolo II 132, Fisciano, 84084 Italy.

${ }^{42}$ Università di Genova, Via Dodecaneso 33, Genova, 16146 Italy.

${ }^{43}$ Normandie Univ, ENSICAEN, UNICAEN, CNRS/IN2P3, LPC Caen, LPCCAEN, 6 boulevard Maréchal Juin, Caen, 14050 France.

${ }^{44}$ University of Granada, Dpto. de Física Teórica y del Cosmos \& C.A.F.P.E., 18071 Granada, Spain.

${ }^{45}$ NIOZ (Royal Netherlands Institute for Sea Research), PO Box 59, Den Burg, Texel, 1790 AB, the Netherlands.

${ }^{46}$ Tbilisi State University, Department of Physics, 3, Chavchavadze Ave., Tbilisi, 0179 Georgia.

${ }^{47}$ Leiden University, Leiden Institute of Physics, PO Box 9504, Leiden, 2300 RA Netherlands.

${ }^{48}$ National Centre for Nuclear Research, 02-093 Warsaw, Poland.

${ }^{49}$ Institut Universitaire de France, 1 rue Descartes, Paris, 75005 France.

${ }^{50}$ University of Johannesburg, Department Physics, PO Box 524, Auckland Park, 2006 South Africa.

${ }^{51}$ Eberhard Karls Universität Tübingen, Institut für Astronomie und Astrophysik, Sand 1, Tübingen, 72076 Germany.

${ }^{52}$ Università di Pisa, Dipartimento di Fisica, Largo Bruno Pontecorvo 3, Pisa, 56127 Italy.

${ }^{53}$ Laboratoire Univers et Particules de Montpellier, Place Eugène Bataillon - CC 72, Montpellier Cédex 05, 34095 France.

${ }^{54}$ Friedrich-Alexander-Universität Erlangen-Nürnberg, Remeis Sternwarte, Sternwartstraße 7, 96049 Bamberg, Germany.

${ }^{55}$ Université de Haute Alsace, 68100 Mulhouse Cedex, France.

${ }^{56}$ University of Münster, Institut für Kernphysik, Wilhelm-Klemm-Str. 9, Münster, 48149 Germany.

${ }^{57}$ Utrecht University, Department of Physics and Astronomy, PO Box 80000, Utrecht, 3508 TA Netherlands.

${ }^{58}$ INFN, CNAF, v.le C. Berti-Pichat, 6/2, Bologna, 40127 Italy. 